Article

\title{
Vertical Dynamic Deflection Measurement in Concrete Beams with the Microsoft Kinect
}

\author{
Xiaojuan Qi ${ }^{1}$, Derek Lichti ${ }^{1}{ }^{1}$, , Mamdouh El-Badry ${ }^{2}$, Jacky Chow ${ }^{1}$ and Kathleen Ang ${ }^{1}$ \\ 1 Department of Geomatics Engineering, University of Calgary, 2500 University Drive N.W., \\ Calgary, Alberta T2N 1N4, Canada; E-Mails: xiqi@ucalgary.ca (X.Q.); \\ jckchow@ucalgary.ca (J.C.); kdang@ucalgary.ca (K.A.) \\ 2 Department of Civil Engineering, University of Calgary, 2500 University Drive N.W., Calgary, \\ Alberta T2N 1N4, Canada; E-Mail: melbadry@ucalgary.ca \\ * Author to whom correspondence should be addressed; E-Mail: ddlichti@ucalgary.ca; \\ Tel.: +1-403-210-9495; Fax: +1-403-284-1980.
}

Received: 11 December 2013; in revised form: 9 January 2014 / Accepted: 7 February 2014 / Published: 19 February 2014

\begin{abstract}
The Microsoft Kinect is arguably the most popular RGB-D camera currently on the market, partially due to its low cost. It offers many advantages for the measurement of dynamic phenomena since it can directly measure three-dimensional coordinates of objects at video frame rate using a single sensor. This paper presents the results of an investigation into the development of a Microsoft Kinect-based system for measuring the deflection of reinforced concrete beams subjected to cyclic loads. New segmentation methods for object extraction from the Kinect's depth imagery and vertical displacement reconstruction algorithms have been developed and implemented to reconstruct the time-dependent displacement of concrete beams tested in laboratory conditions. The results demonstrate that the amplitude and frequency of the vertical displacements can be reconstructed with submillimetre and milliHz-level precision and accuracy, respectively.
\end{abstract}

Keywords: Microsoft Kinect; quantization error; structural deflection measurement 


\section{Introduction}

Bridge structures are a major component of the civil infrastructure of any country. Like any other structure, bridges are designed and built to be safe against failure and to perform satisfactorily over their service life. However, over the past few decades, bridge infrastructure in many parts of the world has been deteriorating at an alarming rate due to inadequate maintenance, excessive loading, economically driven design and construction practices and adverse environmental conditions. Therefore, structural health monitoring of such crucial infrastructure is important for ensuring both their safety and serviceability over their lifespan. Excessive deformations, particularly deflection under long-term effects [1,2] and repeated moving loads (e.g., due to traffic) is one of the major factors that can adversely affect the serviceability of a bridge structure. Throughout the entire life of a structure deflection must not exceed acceptable limits specified by the design codes and standards. Bazant et al. $[3,4]$ compiled records of excessive deflection for a large number of concrete bridges in different parts of the world. In concrete structures, deflection increases with the reduction in stiffness when cracking of the concrete occurs. Cracking and deflection of concrete bridges can be controlled by providing appropriate amounts of pre-stressing reinforcement during construction [5]. However, when the serviceability of a concrete bridge is compromised by excessive cracking and deflection, a promising new technique to enhance performance and extend the service life of the bridge is to bond fibre- or steel-reinforced polymer sheets to the surfaces of the bridge elements. Prior to application of these sheets to actual bridges, their efficacy must be assessed through controlled laboratory testing in which the deflection of strengthened beam or girder specimens is measured under static monotonic and cyclic fatigue loading.

The accurate measurement of deflection of the laboratory specimens can be achieved with different sensors such as dial gauges, linear-variable differential transformers and laser displacement sensors (LDSs). All, however, suffer limitations: the collection of only one-dimensional data; limited measurement range; the high cost to deploy many sensors across an entire structure; and high potential for the sensors to be damaged during testing.

The effectiveness and high accuracy of remote optical measurement methods such as photogrammetry [6-12] and terrestrial laser scanning [13-15] have been demonstrated. Laser scanning systems are, however, best suited for the measurement of displacements under static loading conditions due to their sequential data capture. Though Detchev et al. [16] demonstrate a digital photogrammetric system for both static and dynamic load test measurement-their experiments were conducted concurrently as those reported herein - the cameras' low acquisition rate limits the loading frequency that can be measured to $1 \mathrm{~Hz}$, whereas $3 \mathrm{~Hz}$ is normally required [17,18].

The relatively recent development of range camera technology has, however, opened the possibility of dynamic load measurements. Lichti et al. [19] reported submillimetre deflection measurement accuracy for concrete beams subjected to static load testing using a time-of-flight range camera. Qi et al. [20] reported submillimetre accuracy from their investigation into time-of-flight range camera measurements of concrete beams subjected to dynamic load testing performed at different loading frequencies.

The Microsoft Kinect is a triangulation-based range camera that has been used for many applications such hand gesture recognition [21-25] and detection of the human body [26]. The 
application of the Microsoft Kinect to structural measurement problems can be considered advantageous for several reasons. First, the Microsoft Kinect can acquire three-dimensional (3D) measurements of extended objects such as concrete beams at video frame rate $(30 \mathrm{~Hz})$. Second, in contrast to bulky laser scanner systems, it is a very compact sensor $(\sim 30.5 \mathrm{~cm} \times 7.6 \mathrm{~cm} \times 6.4 \mathrm{~cm}$ and $1.4 \mathrm{~kg}$ ) so it can be easily handled and deployed. Third, the cost of the Microsoft Kinect is extremely low ( CAD 100) in comparison to terrestrial laser scanners ( CAD 40,000+), time-of-flight range cameras ( CAD 5,000) or even digital SLR cameras ( CAD 400+). In considering these advantages, this paper reports on an investigation into the use of the Microsoft Kinect to measure the vertical deflection of a concrete beam subjected to cyclic loads in a laboratory, which simulates traffic loading on bridges.

The paper begins with a general overview of the Microsoft Kinect sensor in Sections 2 and 3 then describes the mathematical modeling for the measurement of the concrete beam deflection in response to cyclic loading. Section 4 presents the experiment design, data collection and the depth data segmentation and displacement signal reconstruction algorithms. Section 5 reports the results of measuring the deflection of a concrete beam under cyclic loading with the Kinect. These are followed by the conclusions in Section 6.

\section{The Microsoft Kinect Sensor}

The Microsoft Kinect is based on PrimeSense chips and consists of an RGB camera, an infrared (IR) projector and an IR camera as illustrated in Figure 1. It is essentially a stereo vision system that determines depth by triangulation. The projector illuminates the scene with an infrared light speckle pattern generated from a set of diffraction gratings. The reflected speckle pattern is captured by the IR camera and is cross-correlated with a reference image. The reference image is obtained by capturing a plane at a known distance from the camera. The depth of a point in object space is determined by triangulation from the corresponding disparity between conjugate points [27].

Figure 1. The Microsoft Kinect.

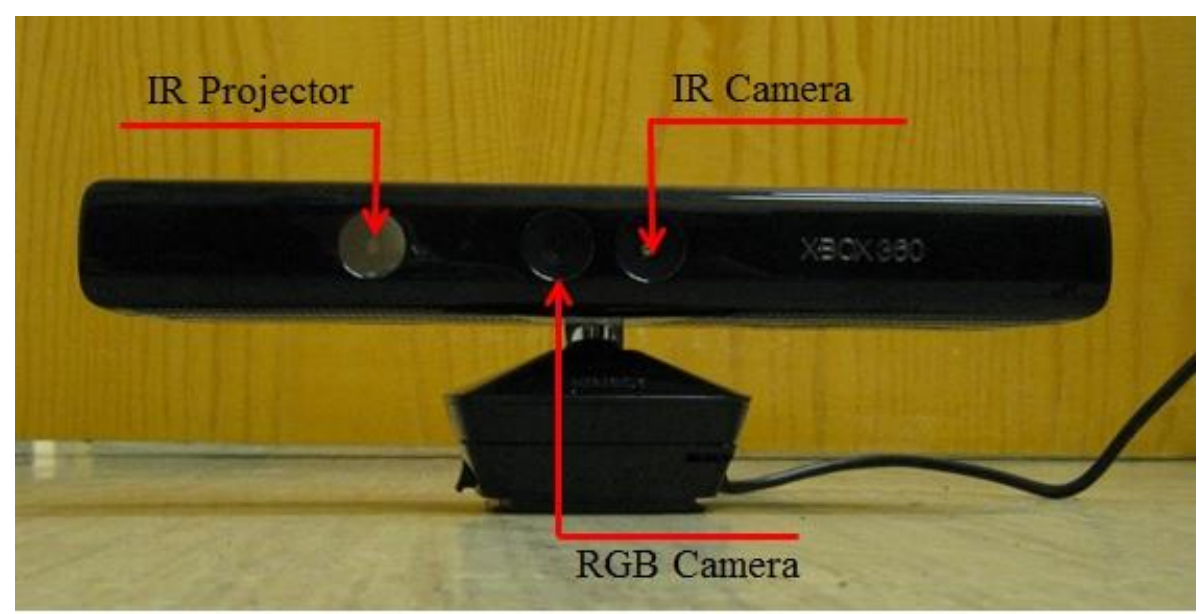

The array size of the IR depth image is $640 \times 480$ pixels, which is smaller than the actual chip size $(1,280 \times 1,024$ pixels $)$ of the IR camera sensor, and has a pixel pitch of $5.2 \mu \mathrm{m}$ and a $6.0 \mathrm{~mm}$ focal-length. The chip size is $6.66 \times 5.33 \mathrm{~mm}^{2}$. The maximum frame rate of the Microsoft Kinect is 
$30 \mathrm{~Hz}$. The depth measurement accuracy of the Microsoft Kinect degrades with increasing distances [28]. The lighting conditions can impact the computation of disparities; for example under strong sunlight, the laser speckles appear with low contrast in the infrared image.

The primary error sources include inadequate calibration and inaccurate disparity measurement. The different optical sensors of the Microsoft Kinect are affected by lens distortions (radial and decentring). In addition, the boresight and leverarms between the cameras may not be properly modeled. Such systematic errors can be reduced by a rigorous calibration procedure, e.g., [29]. The depth measurements of the Microsoft Kinect are derived from the disparities, which are normalized and quantized as 11-bit integers. The effect of disparity measurement quantization on the depth measurement precision, which degrades with the square of the depth, is discussed by Chow and Lichti [29] and is analyzed in detail herein. Asad and Abhayaratne [21] present a method with morphological filtering to reduce the quantization error. A straightforward method to reduce quantization error by spatial averaging many depth measurements is proposed herein.

\section{Deflection Measurement Methods}

\subsection{Three-Dimensional Coordinates from Microsoft Kinect}

According to Khoshelham and Elberink [27], 3D coordinates can be derived from the Microsoft Kinect depth data as follows:

$$
\left\{\begin{array}{c}
X_{k}=-\frac{\text { depth }_{k}}{f}\left(x_{k}-x_{p}+\Delta x\right) \\
Y_{k}=-\frac{\text { depth }_{k}}{f}\left(y_{k}-y_{p}+\Delta y\right) \\
Z_{k}=\text { depth }_{k}
\end{array}\right.
$$

where $\left(x_{k}, y_{k}\right)$ are the image coordinates of the depth image point $k ;\left(x_{p}, y_{p}\right)$ are the coordinates of the principal point; $(\Delta x, \Delta y)$ are correction terms for lens distortions; $\left(X_{k}, Y_{k}, Z_{k}\right)$ are the 3D coordinates of the object point $k ; f$ is focal length of the IR depth camera and $\operatorname{depth}_{k}$ is the depth measurement.

\subsection{Mathematical Model for Concrete Beam Deflection}

Often in fatigue testing the load is applied at a single frequency with a sinusoidal displacement waveform. The measurement objective is to automatically reconstruct the resulting sinusoidal displacement of the loaded beam from sensor data. A time series of depth measurements captured with a Kinect can be used to reconstruct this displacement signal. The displacement can be modeled as follows:

$$
h(t)=C \cos 2 \pi f_{0} t+D \sin 2 \pi f_{0} t+E
$$

where $f_{0}$ is the loading frequency, $C$ and $D$ are the coefficients of the harmonic model and, $E$ is the mean value of the time series.

The first step of our reconstruction algorithm is the initial approximation of some displacement parameters from the spectrum of the depth measurement time series. Although the frame sampling rate of the Microsoft Kinect is nominally uniform, random drop-outs can occur due to the USB data transmission. To overcome the missing data problem, Lomb's method [30] is used to calculate the 
power spectral density (PSD) from the time series. Then, the nominal loading frequency, $f_{0}$ of the sinusoidal motion is identified from the dominant peak (Figure 2).

The false-alarm probability of the time-series, $\alpha$, is calculated to confirm the presence (or the absence) of a periodic signal and to assess the significance of the dominant peak in the PSD. A small value for the false-alarm probability indicates a highly significant periodic signal. If $\alpha$ is less than 0.001 , a highly significant periodic signal exists in the time series and, if $\alpha$ is greater than 0.05 , then the signal is noise [30].

Figure 2. PSD of the depth time series.

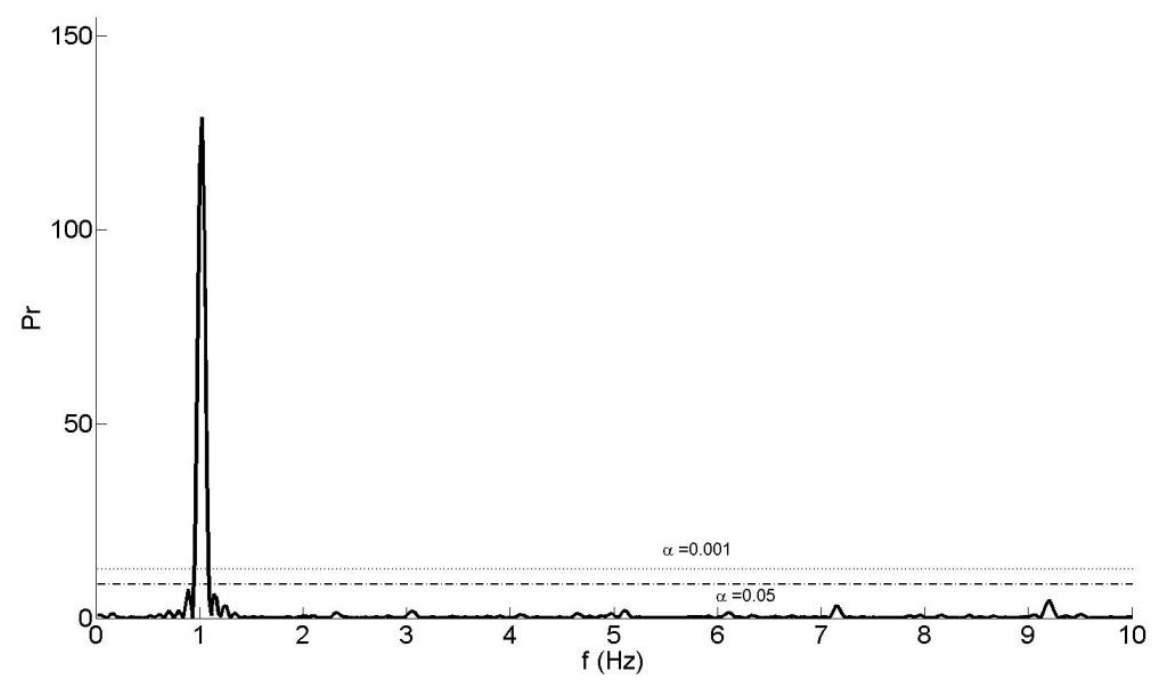

With the approximate loading frequency treated as a constant, $f_{0}$, the coefficients $C, D, E$ are estimated by least-squares under the assumption of a linear functional model. In the final step all four coefficients of the non-linear model are simultaneously estimated by least-squares using the approximate coefficients as initial values for the Taylor series expansion [31]. The amplitude $A$ of the motion, one of the key parameters for the structural analysis, is then derived as:

$$
A=\sqrt{C^{2}+D^{2}}
$$

\section{Experiment and Description}

\subsection{Experiment Design and Data Acquisition}

A $3.3 \mathrm{~m}$ long concrete beam with $150 \mathrm{~mm} \times 300 \mathrm{~mm}$ rectangular cross-section was supported over a $3 \mathrm{~m}$ long span (Figure 3) for the fatigue loading test. The beam was reinforced internally with steel bars and stirrups and externally with a steel fibre reinforced polymer (SRP) sheet bonded to the beam soffit over the entire span. The SRP sheet was used for the purpose of a separate investigation into its efficiency in flexural strengthening of reinforced concrete elements for fatigue resistance. A hydraulic actuator was used to apply a periodically-varying load at two points, each $300 \mathrm{~mm}$ on either side of the concrete beam's mid span, via a 1,400 mm long steel spreader beam.

The experiment comprised two static loading and fatigue loading cycles. The periodic loading cycles - of interest here-were applied to the concrete beam based on load control. First, 36,000 load cycles were applied from $24 \mathrm{kN}$ to $72 \mathrm{kN}$ at $3 \mathrm{~Hz}$ loading frequency causing $2.6 \mathrm{~mm}$ displacement 
amplitude at the mid-span of the beam. In general, fatigue loading tests are conducted at $3 \mathrm{~Hz}[17,18]$. However, in order to meet the sampling frequency requirement of the digital camera system [7], load testing was also conducted at $1 \mathrm{~Hz}$.

Ideally the surface of the concrete beam would be directly measured with the optical sensors. However, nearly $50 \%$ of its top surface at mid-span was occluded by the spreader beam. Thus a targeting means was required to facilitate optical measurement of the concrete beam. As described in [20], the target system comprised thirteen white-washed, thin aluminum witness plates $(220 \mathrm{~mm} \times 50 \mathrm{~mm})$ bonded to the side of the beam at an interval of $250 \mathrm{~mm}$ along its length and numbered 1 to 13 (see Figure 3). The witness plate dimensions were chosen in such a way that the plate would not affect the concrete beam rigidity and hence its deflection. Wider plates, which would be advantageous from an imaging perspective, were ruled out as they would also interact with the cracks in the beam, delaying their formation and restraining their widths. The end result would be a stiffer beam with less deflection.

Figure 3. Photographic image of the experiment setup.

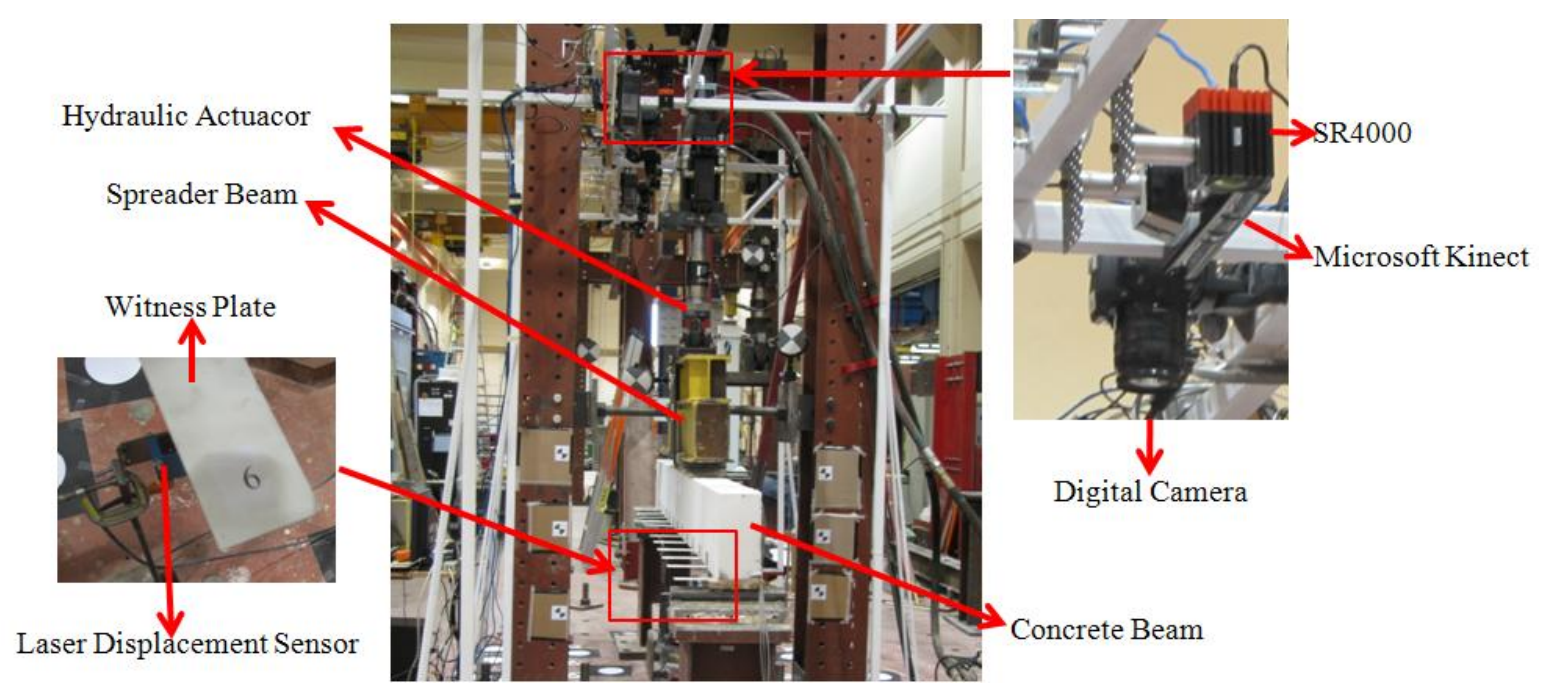

The measurement systems used for the experiment included one Microsoft Kinect, three SR4000 time-of-flight range cameras, a photogrammetric system comprised a set of eight digital cameras and two projectors, and five LDSs. Only the Microsoft Kinect results are presented and analyzed here. Qi and Lichti [20] report the results of the deflection measurements obtained with the SR4000 range cameras while [16] present the photogrammetric system results.

All sensors were mounted on a rigid scaffold assembly approximately $1.9 \mathrm{~m}$ above the top surface of the concrete beam. At this depth the Microsoft Kinect depth precision, which varies inversely with depth squared, is just under $10 \mathrm{~mm}$ [32]. The Microsoft Kinect was warmed up for one hour prior to the fatigue loading test in order to obtain stable measurement data. Chow et al. [33] recommend that at least one hour warm-up is necessary to obtain stable depth measurements. Since only relative displacement measurements were required and the beam displacements were small ( $8 \mathrm{~mm}$ peak-to-peak), the geometric calibration of the Kinect was not required. As shown in [19], subtraction of acquired depth measurements from a zero-load reference has the effect of removing any biases due to un-modeled systematic errors. Many $5 \mathrm{~s}$ long Kinect datasets were collected at the $30 \mathrm{~Hz}$ acquisition 
rate throughout the testing regime. For each loading frequency, five datasets were analyzed, each one being randomly selected from one of the five days of load testing. The elapsed time between the first and last $1 \mathrm{~Hz}$ and $3 \mathrm{~Hz}$ datasets was $55 \mathrm{~min}$ and $2 \mathrm{~h} 28 \mathrm{~min}$, respectively.

The accuracy of the Microsoft Kinect was assessed by comparison with the measurements from KEYENCE LKG407 CCD LDSs. The manufacturer stated linearity of this sensor is $0.05 \%$ of the $100 \mathrm{~mm}$ measurement range and the precision is $2 \mu \mathrm{m}$. Five LDSs were placed under the centroid of thin plates along the length of the beam. Data were acquired with these active triangulation systems at $300 \mathrm{~Hz}$ at the same time as the Kinect to permit direct comparison of the reconstructed displacements.

\subsection{Depth Data Extraction}

Although the Microsoft Kinect provides both depth and RGB images, only the depth image (Figure 4a) was used to extract the witness plates in order to overcome the obstacle of the different fields-of-view of the RGB and IR cameras. For each image in an acquired time series, depth-based segmentation [20] was performed to remove the floor and objects above the witness plates. The resulting binary image is shown in Figure 4b. Second, the eccentricity [34] of the connected regions in the binary image was used to distinguish the thin plates (Figure 4c) from unwanted regions. One witness plate at the end of the beam was excluded by the algorithm due to segmentation errors caused by depth measurements from the beam support. This was not a problem in the ensuing structural analysis since there was little or no motion at the end of the beam. In the last step, image erosion was used to remove the edges of the witness plates (Figure 4d). The 3D centroid of each witness plate region was then calculated and used for the displacement signal reconstruction. Any differences in vertical displacement between the edges of the witness plates are eliminated by the spatial averaging operation.

Figure 4. (a) Depth image from Microsoft Kinect, (b) The binary image after depth-based segmentation, (c) The binary image after eccentricity analysis, and (d) The final result after image erosion.

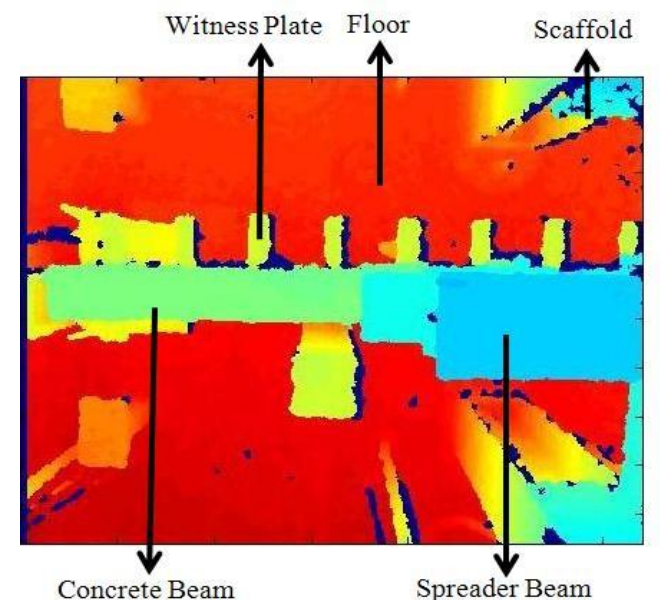

(a)

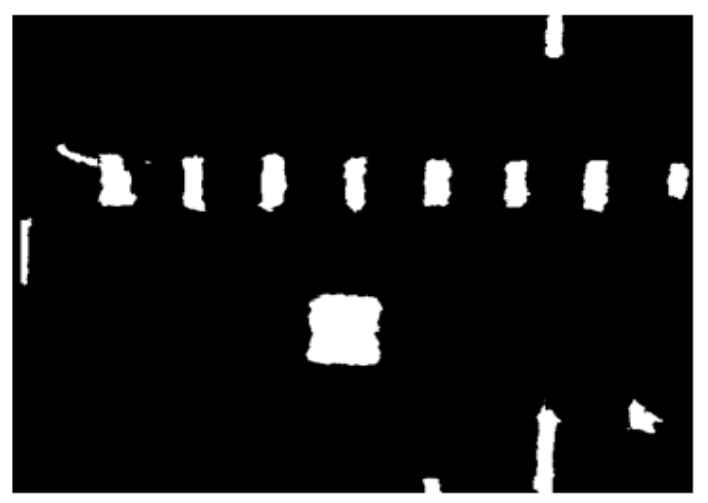

(b) 
Figure 4. Cont.

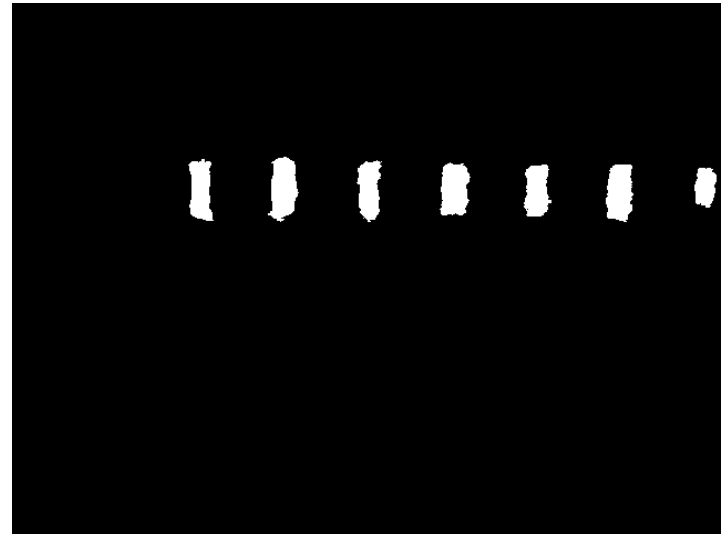

(c)

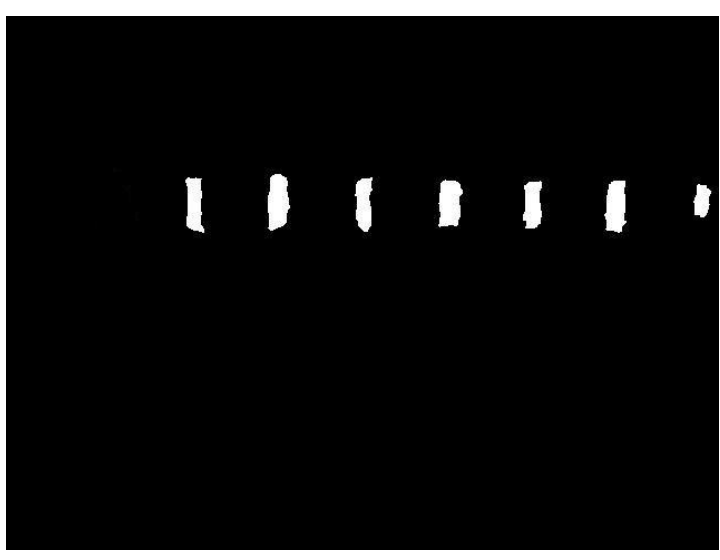

(d)

\section{Results and Analysis}

\subsection{Quantization Error Analysis}

Since the depth measurement accuracy is strongly influenced by the Microsoft Kinect's inherent disparity quantization [27], an analysis of the quantization error effects on the vertical deflection reconstruction was conducted. Figure 5 shows the time-series of the raw depth measurement of a witness plate at the centroid pixel location, while Figure 6 shows the time series of the computed centroid depth using the algorithm described in Section 4.2. The quantization error effects are clearly visible in Figure 5 as the $10 \mathrm{~mm}$ steps in depth and the sinusoidal witness plate motion cannot be inferred from the time series. In Figure 6, however, the effect of the spatial averaging of depth values over the witness plate region is evident as the sinusoidal motion $(\sim 6 \mathrm{~mm}$ peak-to-peak displacement in this example) is visible. High-precision displacement estimates can be expected since the spatial averaging reduces the theoretical quantization error standard deviation [35] as follows:

$$
\sigma_{z}=\frac{\Delta}{\sqrt{12} \sqrt{N}}
$$

where $\Delta$ is the quantization step in depth and $N$ is the number of spatially-averaged depth samples. In this example with $\Delta=10 \mathrm{~mm}$, the depth precision is improved from $2.89 \mathrm{~mm}(N=1)$ to $0.11 \mathrm{~mm}$ $(N=700)$ by averaging the depth measurements within the witness plate region. Even with the spatial averaging, a $10 \mathrm{~mm}$ discontinuity still exists at $4.1 \mathrm{~s}$. This can be easily identified by differentiating the time series and locating the peak. Only data between such discontinuities are then utilized in the displacement signal reconstruction. The luxury of this technique is afforded by the fact that the quantization step is larger than the beam displacement. Though use of a larger plate would provide more samples for the spatial averaging and improve data quality even further, this was not possible for the reasons discussed in Section 4.1. 
Figure 5. Raw Microsoft Kinect depth measurement at the witness plate centroid.

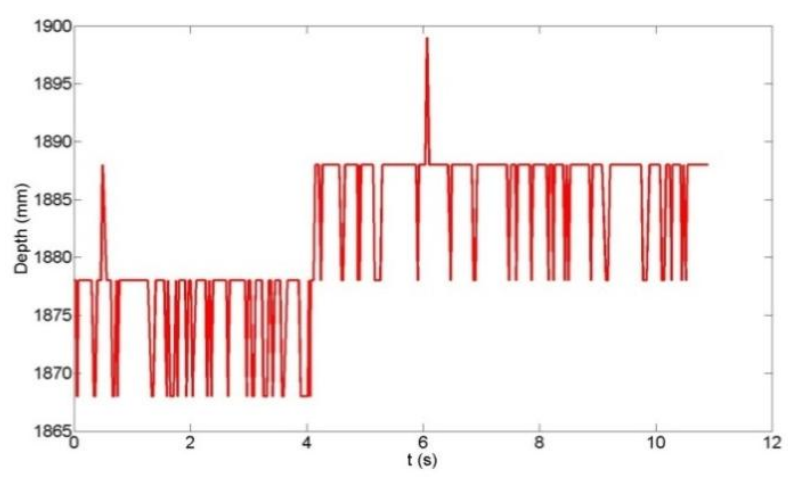

Figure 6. Computed witness plate centroid depth measurement.

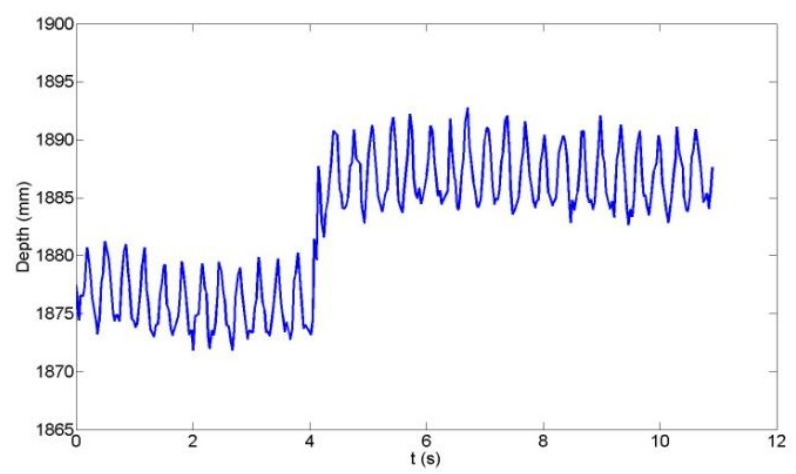

\subsection{Reconstructed Concrete Beam Deflection Results}

Following the previously-outlined procedure, the results achieved for the reconstruction of the vertical displacement of three plates $(3,5$ and 7; plate 7 was located at the mid-span of the concrete beam; plate 3 was near the end) from the Microsoft Kinect data are analyzed for both $1 \mathrm{~Hz}$ and $3 \mathrm{~Hz}$ loading frequencies. LDS data were available at these three plates. Figures 7 and 8 are typical examples of the observed and reconstructed witness plate centroid trajectories with a $1 \mathrm{~Hz}$ nominal loading frequency. Table 1 presents the recovered amplitudes and loading frequencies derived from Microsoft Kinect depth data measurement for the five different datasets, their estimated differences with the LDS (accuracy measures $\Delta \mathrm{A}$ and $\Delta f_{0}$ ) and the estimated precision measures. As can be seen, sub-millimetre amplitude precision and accuracy were achieved in all but one case (dataset 2, plate 5). The estimated loading frequency precision and absolute accuracy were on the order of a few $\mathrm{mHz}$ with only two exceptions.

Figure 7. Kinect observed and reconstructed vertical displacements $\left(f_{0}=1 \mathrm{~Hz}\right)$, dataset 4 witness plate 7 .

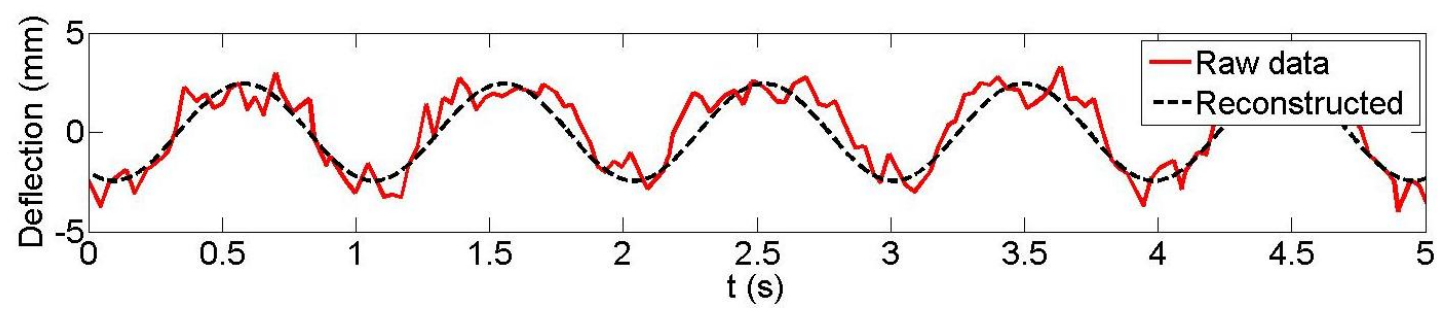


Figure 8. Kinect observed and reconstructed vertical displacements $\left(f_{0}=1 \mathrm{~Hz}\right)$, dataset 5 witness plate 7 .

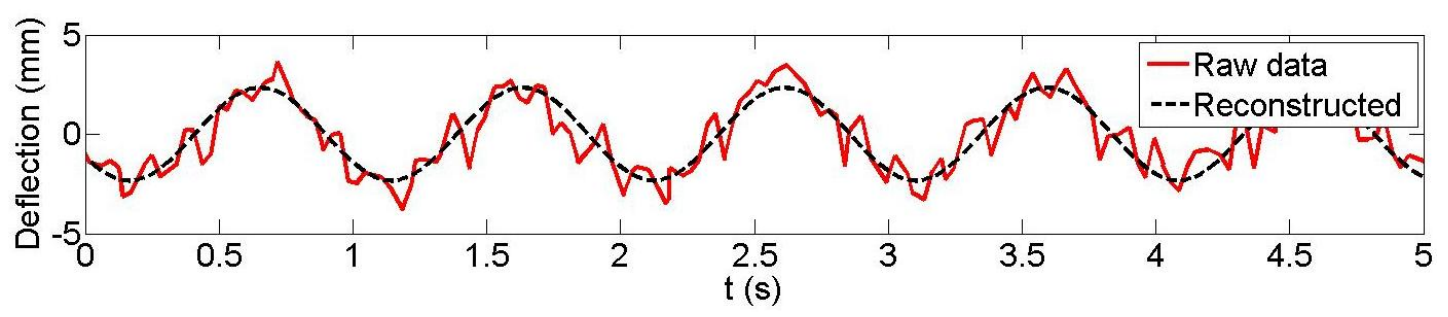

Table 1. Recovered loading frequencies and amplitudes for Plates 3,5 and $7\left(f_{0}=1 \mathrm{~Hz}\right)$. $A_{K I N}, f_{0-K I N}, \sigma_{A-K I N}, \sigma_{f_{0-K I N}}$ and $\sigma_{\hat{r}-K I N}$ are the amplitude, loading frequency and their corresponding standard deviations estimated from the Kinect data; $A_{L D S}, f_{0-L D S}, \sigma_{A-L D S}$ and $\sigma_{f_{0-L D S}}$ are the amplitude, loading frequency and their corresponding standard deviations estimated from the LDS data.

\begin{tabular}{|c|c|c|c|c|c|c|c|c|c|c|}
\hline Plate \# & Set \# & $\begin{array}{l}A_{K I N} \\
(\mathbf{m m}) \\
\end{array}$ & $\begin{array}{l}A_{L D S} \\
(\mathbf{m m})\end{array}$ & $\begin{array}{c}f_{0-K I N} \\
(\mathbf{H z}) \\
\end{array}$ & $\begin{array}{c}f_{0-L D S} \\
(\mathbf{H z})\end{array}$ & $\begin{array}{c}\Delta A \\
(\mathbf{m m}) \\
\end{array}$ & $\begin{array}{c}\Delta \boldsymbol{f}_{0} \\
(\mathbf{m m})\end{array}$ & $\begin{array}{c}\sigma_{A-K I N} \\
(\mathbf{m m}) \\
\end{array}$ & $\begin{array}{c}\sigma_{f_{0-K I N}} \\
(\mathbf{m m})\end{array}$ & $\begin{array}{c}\sigma_{\hat{r}-K I N} \\
(\mathbf{m m}) \\
\end{array}$ \\
\hline \multirow{5}{*}{3} & 1 & 0.72 & 1.12 & 0.9125 & 1.0222 & -0.40 & -0.1097 & 0.17 & 0.0320 & 1.26 \\
\hline & 2 & 1.34 & 1.20 & 1.0192 & 1.0223 & 0.14 & -0.0031 & 0.09 & 0.0123 & 0.59 \\
\hline & 3 & 1.67 & 1.22 & 1.0276 & 1.0224 & 0.45 & 0.0052 & 0.07 & 0.0040 & 0.67 \\
\hline & 4 & 1.54 & 1.21 & 1.0239 & 1.0222 & 0.33 & 0.0017 & 0.05 & 0.0031 & 0.48 \\
\hline & 5 & 1.99 & 1.21 & 1.0231 & 1.0222 & 0.78 & 0.0009 & 0.05 & 0.0012 & 0.63 \\
\hline \multirow{5}{*}{5} & 1 & 2.11 & 2.22 & 1.0240 & 1.0222 & -0.11 & 0.0018 & 0.12 & 0.0083 & 0.95 \\
\hline & 2 & 0.65 & 2.28 & 1.0039 & 1.0222 & -1.63 & -0.0183 & 0.09 & 0.0272 & 0.62 \\
\hline & 3 & 1.51 & 2.28 & 1.0266 & 1.0224 & -0.77 & 0.0042 & 0.11 & 0.0148 & 0.76 \\
\hline & 4 & 2.23 & 2.30 & 1.0252 & 1.0222 & -0.07 & 0.0030 & 0.08 & 0.0036 & 0.75 \\
\hline & 5 & 1.60 & 2.30 & 1.0220 & 1.0222 & -0.70 & -0.0002 & 0.05 & 0.0018 & 0.69 \\
\hline \multirow{5}{*}{7} & 1 & 3.02 & 2.54 & 1.0311 & 1.0222 & 0.48 & 0.0089 & 0.13 & 0.0057 & 1.51 \\
\hline & 2 & 2.66 & 2.57 & 1.0207 & 1.0223 & 0.09 & -0.0016 & 0.11 & 0.0036 & 0.67 \\
\hline & 3 & 2.48 & 2.59 & 1.0284 & 1.0224 & -0.11 & 0.0060 & 0.10 & 0.0041 & 0.91 \\
\hline & 4 & 3.36 & 2.61 & 1.0214 & 1.0222 & 0.75 & -0.0008 & 0.08 & 0.0012 & 1.12 \\
\hline & 5 & 2.33 & 2.61 & 1.0209 & 1.0222 & -0.28 & -0.0013 & 0.08 & 0.0017 & 0.83 \\
\hline
\end{tabular}

Figures 9 and 10 depict the observed and reconstructed witness plate centroid trajectories with $3 \mathrm{~Hz}$ nominal loading frequency. Table 2 presents the recovered loading frequencies and amplitudes derived from the Microsoft Kinect data, their estimated differences with the LDS and the estimated precision measures. The amplitude precisions and differences are of similar magnitudes to those of the $1 \mathrm{~Hz}$ loading frequency results. Additionally, the loading frequency differences are on the order of a few $\mathrm{mHz}$ or less and with only one exception in this case. 
Figure 9. Kinect observed and reconstructed vertical displacements $\left(f_{0}=3 \mathrm{~Hz}\right)$, dataset 2 witness plate 7 .

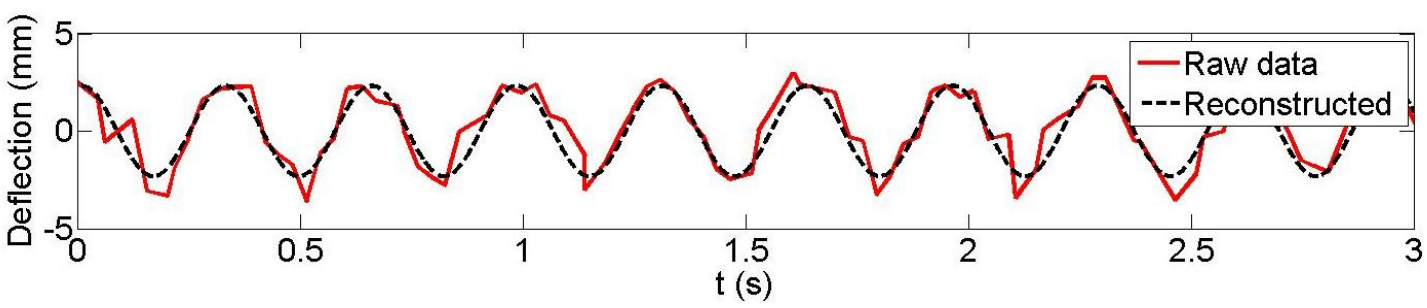

Figure 10. Kinect observed and reconstructed vertical displacements $\left(f_{0}=3 \mathrm{~Hz}\right)$, dataset 5 witness plate 7 .

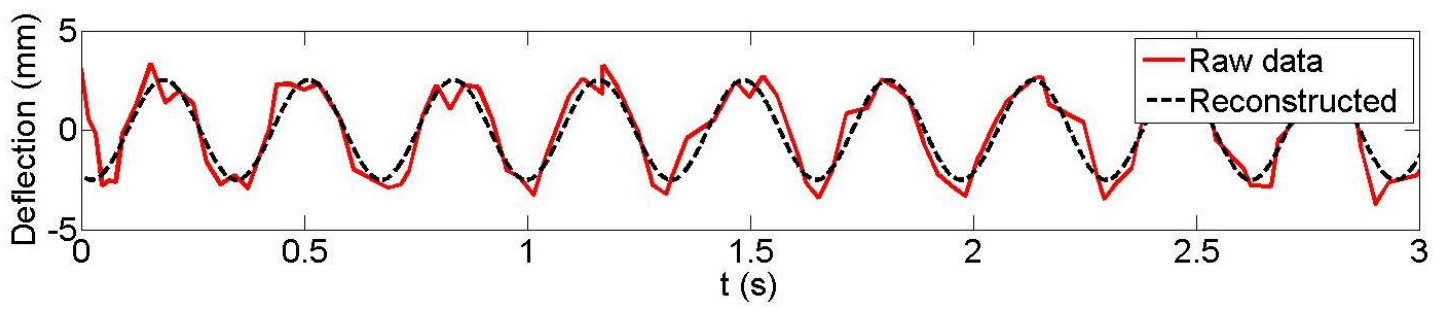

Table 2. Recovered loading frequencies and amplitudes for Plates 3,5 and $7\left(f_{0}=3 \mathrm{~Hz}\right)$. $A_{K I N}, f_{0-K I N}, \sigma_{A-K I N}, \sigma_{f_{0-K I N}}$ and $\sigma_{\hat{r}-K I N}$ are the amplitude, loading frequency and their corresponding standard deviations estimated from the Kinect data; $A_{L D S}, f_{0-L D S}, \sigma_{A-L D S}$ and $\sigma_{f_{0-L D S}}$ are the amplitude, loading frequency and their corresponding standard deviations estimated from the LDS data.

\begin{tabular}{|c|c|c|c|c|c|c|c|c|c|c|}
\hline Plate \# & Set \# & $\begin{array}{l}A_{K I N} \\
(\mathrm{~mm})\end{array}$ & $\begin{array}{l}A_{L D S} \\
(\mathbf{m m})\end{array}$ & $\begin{array}{c}\boldsymbol{f}_{0-K I N} \\
(\mathrm{~Hz})\end{array}$ & $\begin{array}{c}\boldsymbol{f}_{0-L D S} \\
(\mathbf{H z})\end{array}$ & $\begin{array}{c}\Delta A \\
(\mathbf{m m})\end{array}$ & $\begin{array}{c}\Delta \boldsymbol{f}_{0} \\
(\mathbf{m m})\end{array}$ & $\begin{array}{c}\sigma_{A-K I N} \\
(\mathbf{m m}) \\
\end{array}$ & $\begin{array}{c}\sigma_{f_{0-K I N}} \\
(\mathbf{m m})\end{array}$ & $\begin{array}{c}\sigma_{\hat{r}-K I N} \\
(\mathbf{m m})\end{array}$ \\
\hline \multirow{5}{*}{3} & 1 & 1.45 & 1.22 & 3.0653 & 3.0686 & 0.23 & -0.0033 & 0.06 & 0.0039 & 0.57 \\
\hline & 2 & 0.77 & 1.20 & 3.0670 & 3.0680 & -0.43 & -0.0010 & 0.08 & 0.0183 & 0.51 \\
\hline & 3 & 1.94 & 1.20 & 3.0735 & 3.0680 & 0.74 & 0.0055 & 0.11 & 0.0098 & 0.81 \\
\hline & 4 & 1.49 & 1.20 & 3.0666 & 3.0675 & 0.29 & -0.0009 & 0.04 & 0.0011 & 0.67 \\
\hline & 5 & 1.29 & 1.27 & 3.0597 & 3.0675 & 0.02 & -0.0078 & 0.06 & 0.0066 & 0.48 \\
\hline \multirow{5}{*}{5} & 1 & 1.56 & 2.31 & 3.0565 & 3.0686 & -0.75 & -0.0121 & 0.07 & 0.0080 & 0.45 \\
\hline & 2 & 1.32 & 2.33 & 3.0467 & 3.0675 & -1.01 & -0.0208 & 0.17 & 0.0237 & 1.12 \\
\hline & 3 & 1.60 & 2.33 & 3.0926 & 3.0675 & -0.73 & 0.0251 & 0.10 & 0.0102 & 0.70 \\
\hline & 4 & 2.13 & 2.33 & 3.0666 & 3.0675 & -0.20 & -0.0009 & 0.05 & 0.0009 & 0.83 \\
\hline & 5 & 2.29 & 2.34 & 3.0590 & 3.0675 & -0.05 & -0.0085 & 0.12 & 0.0072 & 0.94 \\
\hline \multirow{5}{*}{7} & 1 & 2.18 & 2.54 & 3.0709 & 3.0686 & -0.36 & 0.0023 & 0.13 & 0.0057 & 0.63 \\
\hline & 2 & 2.33 & 2.62 & 3.0665 & 3.0679 & -0.29 & -0.0014 & 0.10 & 0.0045 & 0.82 \\
\hline & 3 & 2.51 & 2.62 & 3.0766 & 3.0680 & -0.11 & 0.0086 & 0.14 & 0.0094 & 1.05 \\
\hline & 4 & 2.04 & 2.65 & 3.0663 & 3.0675 & -0.61 & -0.0012 & 0.06 & 0.0008 & 0.70 \\
\hline & 5 & 2.81 & 2.64 & 3.0660 & 3.0675 & 0.17 & -0.0015 & 0.09 & 0.0022 & 1.13 \\
\hline
\end{tabular}




\subsection{Discussion}

The best-case displacement amplitude accuracy achieved was $0.05 \mathrm{~mm}$ and, although the worst-case amplitude accuracy of $-1.63 \mathrm{~mm}$ represents a significant proportion of the displacement amplitude, the results are much improved over the previously-reported centimetre-level depth precision [32]. The few inaccurate amplitudes and frequencies can be attributed to residual quantization errors. The results demonstrate that small displacement measurements can be made with sub-millimetre accuracy with the Microsoft Kinect by using straightforward spatial filtering and modelling techniques. However, they are not as accurate as what can be achieved with the SR4000 time-of-flight camera [20]. The results presented are independent of the loading frequency, though only two $(1 \mathrm{~Hz}$ and $3 \mathrm{~Hz})$ were tested and they are well below the $15 \mathrm{~Hz}$ Nyquist frequency of the Microsoft Kinect. The missing data problem was only a minor obstacle for the initial stage of the signal reconstruction; it was not an issue for the final signal reconstruction since uniformly-sampled data were not required.

\section{Conclusions and Future Work}

The Microsoft Kinect has been successfully used to measure the periodic vertical displacement of a concrete beam during cyclic loading tests. Automated algorithms for witness plate extraction, spatial data filtering and signal reconstruction were developed for the experiments. The principal advantages of the Microsoft Kinect for structural measurements include: the sensor's wide field-of-view (allowing imaging of a large area — half of the concrete beam — unlike the more accurate LDS point measurement device); the use of straightforward filtering and modelling techniques that allowed sub-millimetre displacements to be measured from a sensor for which the nominal accuracy is $10 \mathrm{~mm}$ at the $2 \mathrm{~m}$ stand-off distance of the experiments.

In this paper only vertical displacements-which were of primary interest-were reported. Future work will concentrate on the extraction of three-dimensional displacement data, which can have value for assessing load eccentricity. This will require improvements to the segmentation algorithm in order to produce more accurate witness plate regions. Cues derived from the RGB imagery will likely be beneficial to this process, but this will require proper registration with the depth imagery after calibration [29]. Further work is also needed to overcome the residual quantization errors that were encountered in a couple of the datasets. Future work should also concentrate on more general, multi-frequency loading conditions since the case of only a single unknown loading frequency has been investigated here.

\section{Acknowledgments}

The authors acknowledge the funding support of the Natural Sciences and Engineering Research Council of Canada (NSERC) and the Canada Foundation for Innovation (CFI). The authors would like to thank Ivan Detchev, Ting On Chan, Hervé Lahamy, Sherif Ibrahim El-Halawany and Vahid Mojarradbahreh for their help during the experiments. The authors are also grateful to Terry Quinn, Dan Tilleman, Mirsad Beric, Daniel Larson and other technical staff of the Structures Laboratory for their help in fabricating the concrete beam specimens and conducting the experiments. 


\section{Author Contributions}

The work presented in this paper is the result of a collaborative effort by all the authors. Xiaojuan Qi developed software for the data collection and data processing and performed the Kinect data acquisition and processing. Derek Lichti contributed to the experiment design and data processing algorithms. Mamdouh El-Badry designed the structural engineering aspects of the study. Jacky Chow contributed to the analyses of the Kinect data. Kathleen Ang contributed to the data acquisition software development and assisted with the data acquisition. All authors contributed to the final manuscript.

\section{Conflicts of Interest}

The authors declare no conflicts of interest.

\section{References}

1. El-Badry, M.; Ghali, A.; Megally, S. Deflection prediction of concrete bridges: Analysis and field measurements on a long-span bridge. Struct. Concr. 2001, 2, 1-14.

2. Ghali, A.; Elbadry, M.; Megally, S. Two-year deflections of the Confederation Bridge. Can. J. Civ. Eng. 2000, 6, 1139-1149.

3. Bazant, Z.P.; Huber, M.H.; Yu, Q. Excessive deflections of record-span prestressed box girders: Lessons learned from collapse of the Koror-Babeldoab Bridge in Palau. Concr. Int. 2010, 6, 45-52.

4. Bazant, Z.P.; Huber, M.H.; Yu, Q. Perverseness of excessive segmental bridge deflections: Wake-up call for creep. ACI Struct. J. 2011, 6, 766-774.

5. El-Badry, M.; Ghali, A.; Gayed, R. Deflection control of prestressed box girder bridges. J. Bridge Eng., in press.

6. Barazzetti, L.; Scaioni, M. Development and implementation of image-based algorithms for measurement of deformations in material testing. Sensors 2010, 10, 7469-7495.

7. Detchev, I.; Habib, A.; El-Badry, M. Image-based Deformation Monitoring of Statically and Dynamically Loaded Beams. In Proceedings of the International Achieves of the Photogrammetry, Remote Sensing and Spatial Information Sciences, Melbourne, Australia, 25 August-1 September 2012; Volume XXXIX-B5, p. 6.

8. Fraser, C.; Riedel, B. Monitoring the thermal deformation of steel beams via vision metrology. ISPRS J. Photogramm. Remote Sens. 2000, 55, 1-10.

9. Kwak, E.; Detchev, I.; Habib, A.; El-Badry, M.; Hughes, C. Precise photogrammetric reconstruction using model-based image fitting for 3D beam deformation monitoring. ASCE J. Surv. Eng. 2013, 3, 143-155.

10. Maas, H.; Hampel, U. Photogrammetric techniques in civil engineering material testing and structure monitoring. Photogramm. Eng. Remote Sens. 2006, 1, 39-45.

11. Whiteman, T.; Lichti, D.; Chandler, I. Measurement of Deflections in Concrete Beams by Close-Range Digital Photogrammetry. In Proceedings of the Joint International Symposium on Geospatial Theory, Processing and Applications, Ottawa, Canada, 2002; p. 9. 
12. Ye, J.; Fu, G.; Poudel, U. Edge-based close-range digital photogrammetry structural deformation measurement. J. Eng. Mech. 2011, 7, 475-483.

13. Gordon, S.; Lichti, D. Modeling terrestrial laser scanner data for precise structural deformation measurement. ASCE J. Surv. Eng. 2007, 2, 72-80.

14. Park, H.; Lee, H. A new approach for health monitoring of structures-terrestrial laser scanning. Comput. Aided Civ. Infrastruct. Eng. 2007, 1, 19-30.

15. Rönnholm, P.; Nuikka, M.; Suominen, A.; Salo, P.; Hyyppä, H.; Pöntinen, P.; Haggrén, H.; Vermeer, M.; Puttonen, J.; Hirsi, H.; et al. Comparison of measurement techniques and static theory applied to concrete beam deformation. Photogramm. Rec. 2009, 128, 351-371.

16. Detchev, I.; Habib, A.; El-Badry, M. Dynamic beam deformation measurements with off-the-shelf digital cameras. J. Appl. Geod. 2013, 3, 147-157.

17. Heffernan, P.J.; Erki, M.A. Fatigue behavior of reinforced concrete beams strengthened with carbon fiber reinfoced plastic lamniates. J. Compos. Constr. 2004, 2, 132-140.

18. Papakonstantinou, C.G.; Petrou, M.F.; Harries, K.A. Fatigue behavior of RC beams strengthened with GFRP sheets. J. Compos. Constr. 2001, 4, 246-253.

19. Lichti, D.D.; Jamtsho, S.; El-Halawany, I.S.; Lahamy, H.; Chow, J.; Chang, T.O.; El-Badry, M. Structural deflection measurement with a range camera. ASCE J. Surv. Eng. 2012, 2, 66-76.

20. Qi, X.; Lichti, D.D.; El-Badry, M.; Chan, T.O.; El-Halawany, S.; Lahamy, H.; Steward, J. Structural dynamic deflection measurement with range cameras. Photogramm. Rec. in press.

21. Asad, M.; Abhayaratne, C. Kinect Depth Stream Pre-Processing for Hand Gesture Recognition. In Proceedings of the IEEE International Conference on Image Processing, Melbourne, Australia, 15-18 September 2012; pp. 3735-3739.

22. Li, Y. Hand gesture recognition using Kinect. In Proceedings of the 2012 IEEE 3rd International Conference on Software Engineering and Service Science (ICSESS), Beijing, China, 22-24 June 2012; pp. 196-199.

23. Li, Y. Multi-scenario Gesture Recognition Using Kinect. In Proceedings of the 2012 17th International Conference on Computer Games (CGAMES), 30 July 30-1 August 2012; pp. 126-130.

24. Ren, Z.; Yuan, J.; Meng, J.; Zhang, Z. Robust part-based hand gesture recognition using kinect sensor. IEEE Trans. Multimed. 2013, 5, 1110-1120.

25. Lahamy, H.; Lichti, D.D. Towards real-time and rotation-invariant American Sign Language alphabet recognition using a range camera. Sensors 2012, 12, 14416-14441.

26. Xia, L.; Chen, C.; Aggarwal, J.K. Human Detection Using Depth Information by Kinect. In Proceedings 2011 IEEE Computer Society Conference on Computer Vision and Pattern Recognition Workshops (CVPRW), 20-25 June 2011; pp. 20-25.

27. Khoshelham, K.; Elberink, S. Accuracy and resolution of kinect depth data for indoor mapping applications. Sensors 2012, 12, 1437-1454.

28. Langmann, B.; Hartmann, K.; Loffeld, O. Depth Camera Technology Comparison and Performance Evaluation. In Proceedings of the 1st Internationl Conference on Pattern Recognition Applications and Methods, Vilamoura, Portugal, 6-8 February 2012; pp. 438-444.

29. Chow, J.C.K.; Lichti, D.D. Photogrammetric bundle adjustment with self-calibration of the PrimeSense 3D camera technology: Microsoft Kinect. IEEE Access 2013, 1, 465-474. 
30. Press, H.W.; Teukolsky, A.S.; Vetterling, T.W.; Flannery, P.B. Numerical Recipes in C, 2nd ed.; Cambridge University Press: New York, NY, USA, 1992.

31. Cheney, W.; Kincaid, D. Numerical Mathematics and Computing, 6th ed.; Thompson Brooks/Coles: Belmont, CA, USA, 2007.

32. Khoshelham, K. Accuracy Analysis of Kinect Depth Data. In Proceedings of the International Archives of the Photogrammetry, Remote Sensing and Spatial Information Sciences, ISPRS Calgary 2011 Workshop, Calgary, Canada, 29-31 August 2011; Volume XXXVIII-5/W12, pp. 133-138.

33. Chow, J.C.K.; Ang, K.; Lichti, D.; Teskey, W. Performance Analysis of a Low Cost Triangulation-Based 3D Camera: Microsoft Kinect System. In Proceedings of the International Achieves of the Photogrammetry, Remote Sensing and Spatial Information Sciences, Melbourne, Australia, 25 August-1 September 2012; Volume XXXIX-B5, pp. 239-244.

34. Rohs, M. Real-world interaction with camera phones. Lect. Notes Comput. Sci. 2005, 3598, 74-89.

35. Oppenheim, A.V.; Schafer, R.W. Discrete-Time Signal Processing; Prentice Hall: Englewood Cliffs, NJ, USA, 1989.

(C) 2014 by the authors; licensee MDPI, Basel, Switzerland. This article is an open access article distributed under the terms and conditions of the Creative Commons Attribution license (http://creativecommons.org/licenses/by/3.0/). 